\title{
REGULARITY IN TERMS OF REDUCTIONS IN LOCAL NOETHER LATTICES
}

\author{
MICHAEL E. DETLEFSEN ${ }^{1}$
}

\begin{abstract}
An $(n, d)$-sequence in a local Noether lattice $(L, M)$ is a sequence of words which satisfy certain factorization properties. If $(L, M)$ satisfies the union condition, there exist $(n, d)$-sequences which can be extended to minimal bases for the powers of $M$. Consequently, if $(L, M)$ satisfies the union condition, $(L, M)$ is regular if and only if $M$ is a minimal reduction.
\end{abstract}

Some time ago, Northcott and Rees [8, pp. 147-152] presented a result characterizing certain regular local Noetherian rings in terms of reductions of the maximal ideal. An ideal $A$ is a reduction of an ideal $B$ if $A \subseteq B$ and $A B^{n}=B^{n+1}$ for some integer $n>0$. This ideal-theoretic definition is easily generalized to Noether lattices. However, the proof of the result in [8] does not generalize since it involves the use of polynomial rings for which, to our knowledge, we have no suitable analog in the theory of Noether lattices.

In this note, we overcome this obstacle by introducing $(n, d)$-sequences, sequences of words in the underlying multiplicative semigroup of a Noether lattice. We show that a local Noether lattice satisfying the union condition is regular if and only if its maximal element is a minimal reduction.

1. $(n, d)$-sequences. Throughout this section $S^{*}=\left\{x_{1}, x_{2}, \cdots, x_{m}, \cdots\right\}$ is a countably infinite set and $S$ is the free monoid on $S^{*}$.

DEFINITION 1.1. If $d \geqq 2$ and $U, V_{1}, \cdots, V_{t}$ are words in $S$, we say that the sequence $V_{1}, \cdots, V_{t}$ is $d$-factorable from $U$ if each $V_{i}$ has at least one of a set of fewer than $d$ factors distinct from the factors of $U$. $W_{1}, W_{2}, \cdots, W_{q}$ is an $(n, d)$-sequence if the $W_{i}$ are all of degree $n$ and $W_{l+1}, \cdots, W_{q}$ is $d$-factorable from $W_{l}$ for $1 \leqq l<q$.

Presented to the Society, March 28, 1972; received by the editors July 18, 1972 and, in revised form, June 7, 1973.

AMS (MOS) subject classifications (1970). Primary 13A15, 13H05, 13E05; Secondary 06A30, $13 \mathrm{H} 15$.

Key words and phrases. Regular local Noether lattice, free monoid, reduction, union condition, analytically independent, $(n, d)$-sequence.

${ }^{1}$ This research was supported in part by NASA Grant NGT 16-001-004. The author wishes to express his appreciation to the referee for his constructive comments and to E. W. Johnson for his guidance in preparation of this result. 
The degree of a word $\prod_{j} x_{j}^{a(j)}$ is $\sum_{j} a(j)$. If $x^{a}$ appears in a word with $a>0$ then $x$ is a factor of that word. We use $C(m, n)$ to denote the binomial coefficient and $|A|$ to denote the cardinality of the set $A$.

When $\Gamma: W_{1}, \cdots, W_{q}$ is an $(n, d)$-sequence consisting of products of principal elements $\left\{E_{i}\right\}$ in a local Noether lattice $L$, the $d$-factorability of $\Gamma$ insures that for $1 \leqq l<q, \bigvee_{i=l+1}^{a} W_{i} \leqq T\left(\bigvee_{i=l+1}^{q} W_{i}^{\prime}\right)$ where $T$ is the join of fewer than $d$ of the $E_{i}$ distinct from the factors of $W_{l}$. This factorization property guarantees in our context that $\bigvee W_{i}$ can be extended to a minimal base for $A^{n}$ where $A=\bigvee E_{i}$ (Lemma 2.5). Moreover, when $A$ is the maximal element of $L$, the existence of $(n, d)$-sequences of length $C(n+d-1, d-1)$ forces minimal bases for $A^{n}$ to be long enough to make $L$ regular (Theorem 2.9). We now demonstrate the existence of such $(n, d)$-sequences.

REMARK 1.2. If $\Gamma: W_{1}, \cdots, W_{q}$ is an $(n, d)$-sequence with factors from $F \subseteq S^{*}$ and $t=x_{k}$ is any element in $S^{*} \backslash F$, then $t^{a} \Gamma: t^{a} W_{1}, \cdots, t^{a} W_{q}$ is an $(n+a, d)$-sequence for any $a \geqq 0$. If $F=\left\{x_{1}, \cdots, x_{m}\right\}$ and $\phi$ is any injection from $\{1,2, \cdots, m\}$ into the nonzero positive integers, then the sequence, denoted $\Gamma_{\phi}$, obtained by substituting $\phi(i)$ for $i, 1 \leqq i \leqq m$, as subscripts in $\Gamma$ is an $(n, d)$-sequence with factors from $F_{\phi}=\left\{x_{\phi(1)}, \cdots\right.$, $\left.x_{\phi(m)}\right\}$. Note that we may assume that $(n, d)$-sequences have factors from $\left\{x_{1}, \cdots, x_{p}\right\}$ for some $p$.

THEOREM 1.3. For all $n \geqq 1$ and for all $d \geqq 2$, there exists an $(n, d)$ sequence of words from $S$ of length $C(n+d-1, d-1)$.

Proof. We proceed by induction on $n$ and $d$. For $n \geqq 1$, the sequence

$$
x_{1}^{n}, x_{n}^{n}, x_{n-1}^{n-1} x_{n}, x_{n-2}^{n-2} x_{n-1} x_{n}, \cdots, x_{2}^{2} x_{3} \cdots x_{n}, x_{1} x_{2} \cdots x_{n}
$$

is an $(n, 2)$-sequence of length $C(n+1,1)$. For $d \geqq 2$, the sequence $x_{1}, x_{2}, \cdots, x_{d}$ is a $(1, d)$-sequence of length $C(d, d-1)$.

By our induction hypothesis, for $d \geqq 3$, let $\Gamma: W_{1}, W_{2}, \cdots, W_{q}$ be an $(n, d)$-sequence with factors from $F=\left\{x_{1}, \cdots, x_{m}\right\}$ of length $q=C(n+d-1, d-1)$ and $\Delta: V_{1}, V_{2}, \cdots, V_{r}$ be an $(n+1, d-1)$-sequence of length $r=C((n+1)+(d-2), d-2)$ with factors from $G=\left\{x_{1}, \cdots, x_{p}\right\}$. If $\phi(i)=p+i, 1 \leqq i \leqq m$, and $t=x_{m+p+1}$, by Remark $1.2, t \Gamma_{\phi}: t W_{1}^{\prime}, t W_{2}^{\prime}, \cdots$, $t W_{q}^{\prime}$ is an $(n+1, d)$-sequence which we write as $V_{r+1}, \cdots, V_{r+\alpha}$.

We claim that the sequence $V_{1}, V_{2}, \cdots, V_{r+q}$ is an $(n+1, d)$-sequence. Clearly, the degree of each $V_{i}$ is $n+1$. If $r+1 \leqq l<r+q$, then since $t \Gamma_{\phi}$ is an $(n+1, d)$-sequence, $V_{l+1}, \cdots, V_{r+q}$ is $d$-factorable from $V_{l}$. Moreover, $t$ is a factor of $V_{r+1}, \cdots, V_{r+q}$ but not a factor of $V_{r}$; so, $V_{r+1}, \cdots, V_{r+q}$ is $d$-factorable from $V_{r}$. Finally, if $1 \leqq l<r$, since $\Delta$ is an $(n+1, d-1)$ sequence, there is a subset $A$ of $F$ such that $|A| \leqq d-2$, each of $V_{l+1}, \cdots, V_{r}$ has some element in $A$ as a factor, and no element in $A$ is a factor of $V_{l}$. 
But then each of $V_{l+1}, \cdots, V_{r+q}$ has some element of $A \cup\{t\}$ as a factor and no element of $A \cup\{t\}$ is a factor of $V_{l}$. Hence, in this case $V_{l+1}, \cdots$, $V_{r+q}$ is $d$-factorable from $V_{l}$. Hence, $V_{1}, \cdots, V_{r+q}$ is an $(n+1, d)$-sequence of length

$$
\begin{aligned}
r+q & =C((n+1)+(d-2), d-2)+C(n+d-1, d-1) \\
& =C((n+1)+(d-1), d-1) . \quad \text { Q.E.D. }
\end{aligned}
$$

Let $L$ be a local Noether lattice and $T^{*}=\left\{E_{i}\right\}$ be a countably infinite set of elements in $L$. Let $T$ be the free commutative monoid on $T^{*}$. By Theorem 1.3, for all $n \geqq 1$ and $d \geqq 2$, there exists an $(n, d)$-sequence of length $C(n+d-1, d-1)$ with factors from $T^{*}$. Reading the juxtaposition multiplication on $T$ as the multiplication in $L$, we obtain

Corollary 1.4. Let $L$ be a local Noether lattice and $T^{*}=\left\{E_{i}\right\}$ be a countably infinite subset of $L$. Then there exists an $(n, d)$-sequence of elements in $L$ of length $C(n+d-1, d-1)$ for all $n \geqq 1$ and for all $d \geqq 2$.

Again, using the technique of reading juxtaposition multiplication as multiplication of the semigroup; and conversely, we have

CoROllary 1.5. Let $L,\left\{E_{i}\right\}$, and $S,\left\{x_{i}\right\}$ be as above. There exists an $(n, d)$-sequence of length $t$ in $L$ with factors from $\left\{E_{1}, \cdots, E_{p}\right\}$ if and only if there exists an $(n, d)$-sequence of length $t$ in $S$ with factors from $\left\{x_{1}, \cdots, x_{p}\right\}$.

2. Reductions and regularity. We now turn to our application of $(n, d)$-sequences to the study of reductions in certain local Noether lattices. Throughout this section $(L, M)$ is a local Noether lattice with maximal element $M$.

Definition 2.1. An element $B$ in $L$ is a reduction of an element, $A$, in $L(B \rho A)$ if $B \leqq A$ and $B A^{n}=A^{n+1}$ for some $n>0$. If $B$ is a reduction of $A$, then $B$ is a minimal reduction of $A$ if $C \leqq B$ and $C \rho A$ implies that $C=B$.

The following theorem is proved as in [8, pp. 146-147] with the appropriate lattice-theoretic interpretations.

THeOREM 2.2. For $A, B$, and $C$ in $L$ :

(1) $C \rho B$ and $B \rho A$ implies $C \rho A$.

(2) $C \rho A$ if and only if $(C \vee A M) \rho A$.

(3) $B \rho A$ implies there exists a minimal reduction $D$ of $A$ such that $D \leqq B$.

(4) If $C$ is a minimal reduction of $A$ and $C \leqq B \leqq A$, then every minimal base for $C$ can be extended to a minimal base for $B$. 
Definition 2.3. $L$ satisfies the union condition if given any finite collection of elements, $A_{1}, A_{2}, \cdots, A_{k}$, in $L$ and any $A$ in $L$ such that $A \pm A_{i}$ for $i=1,2, \cdots, k$, there exists a principal element $B$ in $L$ such that $B \leqq A$ and $B \leq A_{i}$ for $i=1,2, \cdots, k[6,1.7]$.

If $R$ is a local Noetherian ring (commutative with identity) with an infinite residue field, then the lattice of ideals of $R$ satisfies the union condition.

If $B \neq I$ is an element of $L$, let $l(B / B M)$ denote the number of elements in a minimal base for $B$ (which equals the length of the composition series from $B M$ to $B$ [5]). For the remainder of this paper, we assume $L$ satisfies the union condition. The union condition forces each element $B$ in $L$ with $l(B / B M)>1$ to have an infinite number of minimal bases. More precisely

Lemma 2.4. If $B$ is an element of $L$ such that $l(B / B M)=d>1$, then there exists a countably infinite set of principal elements $\left\{E_{i}\right\}$ such that any $d$ of them forms a minimal base for $B$.

Proof. Suppose that $B=E_{1} \vee E_{2} \vee \cdots \vee E_{d}$ where the $E_{i}$ 's form a minimal base and that $E_{1}, E_{2}, \cdots, E_{d+n}$ have been chosen, $n \geqq 0$. Let

$$
\left\{P_{\alpha}\right\}=\left\{\bigvee_{j \in J_{\alpha}} E_{j} \quad B M \mid J_{\alpha} \subseteq\{1, \cdots, d+n\} \text { and }\left|J_{\alpha}\right|=d-1\right\} .
$$

By the union condition, since $B+P_{\alpha}$ for each $\alpha$, and $B \leq B M$, there exists a principal element, $E_{d+n+1} \leqq B$, in $L$ such that $E_{d+n+1} \leq P_{\alpha}$ for each $\alpha$ and $E_{d+n+1} B M$. Since $l(B / B M)=d, E_{d+n+1} \vee\left(\bigvee_{j \in J_{\alpha}} E_{j}\right)$ forms a minimal base for $B$ for each $\alpha$. By induction our proof is complete. Q.E.D.

Lemma 2.5. If $B$ is a minimal reduction of $A$ in $L$ with $l(B / B M)=d>1$ and if $\left\{E_{i}\right\}$ is a countably infinite set of principal elements for $B$ as in Lemma 2.4 , then any $(n, d)$-sequence in the subsemigroup of $L$ generated by the $E_{i}$ can be extended to a minimal base for $A^{n}$.

Proof. First we show that if $T=F_{m 1}^{n(1)} F_{m 2}^{n(2)} \cdots F_{m t}^{n(t)}$ is any product of the $E_{i}$ 's such that $\sum_{j} n(j)=n$, then $T S B^{n-1} \vee A^{n} M$ where $S$ is the join of $d-1$ of the $E_{i}$ 's distinct from the $F_{m j}$. If $T \leqq S B^{n-1} \vee A^{n} M$, then

$$
B^{n} \vee A^{n} M=\left[\left(F_{m 1} \vee S\right)^{n(1)}\left(F_{m 2} \vee S\right)^{n(2)} \cdots\left(F_{m t} \vee S\right)^{n(t)}\right] \vee A^{n} M
$$

since the join of any $d$ of the $E_{i}$ 's is $B$. Then $B^{n} \vee A^{n} M \leqq\left(T \vee S B^{n-1}\right) \vee A^{n} M=$ $S B^{n-1} \vee A^{n} M \leqq B^{n} \vee A^{n} M$. So, if $B A^{r}=A^{r+1}$,

$$
\begin{aligned}
A^{n+r} & =B^{n} A^{r} \leqq B^{n} A^{r} \vee A^{n+r} M=\left(B^{n} \vee A^{n} M\right) A^{r} \\
& =S B^{n-1} A^{r} \vee A^{n+r} M=S A^{n+r-1} \vee A^{n+r} M .
\end{aligned}
$$


Hence, $A^{n+r}=S A^{n+r-1}$ with $S$ strictly less than $B$. This contradicts the fact that $B$ is a minimal reduction of $A$.

Now let $W_{1}, \cdots, W_{a}$ be an $(n, d)$-sequence with factors from $\left\{E_{1}, \cdots, E_{p}\right\}$. Then each $W_{i}$ is a principal element in $L$. If $W_{a} \leqq A^{n} M$, then

$$
W_{q} \leqq\left(E_{p+1} \vee E_{p+2} \vee \cdots \vee E_{p+d-1}\right) B^{n-1} \vee A^{n} M,
$$

contradicting the first paragraph. So $W_{q} A^{n} M$. Suppose that $W_{t} \leqq$ $W_{t+1} \vee \cdots \vee W_{q} \vee A^{n} M$ where $1 \leqq t<q$. Since $W_{t+1}, \cdots, W_{q}$ is $d$-factorable from $W_{t}$, there exists a subset, $\left\{F_{1}, \cdots, F_{k}\right\}$, of $\left\{E_{1}, \cdots, E_{p}\right\}$ with $k \leqq d-1$ such that each of the elements $W_{t+1}, \cdots, W_{q}$ has one of the $F_{i}$ as a factor but no $F_{i}$ is a factor of $W_{t}$. Therefore, $W_{t} \leqq\left(F_{1} \vee \cdots \vee F_{k}\right) B^{n-1} \vee$ $A^{n} M$. Again this is a contradiction. Hence, $W_{1}, \cdots, W_{q}$ can be extended to a minimal base for $A^{n}$. Q.E.D.

Definition 2.6. Principal elements, $E_{1}, \cdots, E_{d}$ in $L$ are analytically independent in $A$ if the join of all products of $E_{1}, \cdots, E_{d}$ of degree $n$ can be extended to a minimal base for $A^{n}$ for all $n \geqq 1$. If $E_{1}, \cdots, E_{d}$ are analytically independent in $E_{1} \vee \cdots \vee E_{d}$, we say that $E_{1}, \cdots, E_{d}$ are analytically independent.

REMARK 2.7. Let $R$ be a commutative local Noetherian ring with identity and maximal ideal $\mathfrak{M}$. Then $t_{1}, \cdots, t_{r}$ in $\mathfrak{M}$ are analytically independent in the sense of [7, p. 67] if and only if the ideals $\left(t_{1}\right), \cdots,\left(t_{r}\right)$ are analytically independent in $L(R)$, the lattice of ideals of $R$, in the sense of Definition 2.6.

Proof. Suppose $\left(t_{1}\right), \cdots,\left(t_{r}\right)$ are not analytically independent in $L(R)$. Then some product $\Pi\left(t_{i}\right)^{\xi(i)}$ of degree, say, $n$ is contained in the join (ideal sum) of different products of the ideals $\left(t_{i}\right)$ of degree $n$ join $\left(t_{1}, \cdots, t_{r}\right)^{n} \mathfrak{M}$. But then $\prod t_{i}^{\xi(i)}$ is equal to $\varphi\left(t_{1}, \cdots, t_{r}\right)$ where $\varphi$ is a form of degree $n$; and $\varphi\left(t_{1}, \cdots, t_{r}\right)-\prod t_{i}^{\xi(i)}$ gives a form of degree $n$ which equals 0 when evaluated at $t_{1}, \cdots, t_{r}$ but all of the coefficients are not in $\mathfrak{M}$.

Conversely, if $\left(t_{1}\right), \cdots,\left(t_{r}\right)$ are analytically independent in $L(R)$ and $\Phi\left(z_{1}, \cdots, z_{r}\right)$ is a form of degree $n$, suppose $\Phi\left(t_{1}, \cdots, t_{r}\right)=0$ and the coefficient $a$ of $\prod t_{i}^{\xi(i)}$ is not in $\mathfrak{M}$. Then $\prod\left(t_{i}\right)^{\xi(i)}$ is contained in the join of different products of the $\left(t_{i}\right)$ of degree $n$ since $(a)=R$ which is a contradiction. Q.E.D.

We agree that 0 in $L$ is analytically independent in $A$ for any $A$ in $L$. Note that $E_{1}, \cdots, E_{d}$ are analytically independent in $A$ if and only if $l\left(\left(B^{n} \vee A^{n} M\right) / A^{n} M\right)=C(n+d-1, d-1)$ for all $n \geqq 1$ where $B=E_{1} \vee \cdots \vee E_{d}$.

Let $Q_{1}, \cdots, Q_{n}$ be a system of parameters for $L$ and let $Q=Q_{1} \vee \cdots$ $\vee Q_{n}$. Then there exists a polynomial $p_{Q}(x)$ with rational coefficients such that $p_{Q}(k)=l\left(Q^{k} / Q^{k} M\right)$ for all sufficiently large $k$. The degree of $p_{Q}(x)$, the Hilbert polynomial for $Q$, is $n-1[5, \S 3]$. 
Let $J_{m}$ be the set of products of the $Q_{i}$ of degree $m$. Let us agree to write the elements of $J_{m}$ as the ordered product, $Q_{1}^{t(1)} \cdots Q_{n}^{t(n)}$ where some of the exponents may be zero. (If $A$ is in $L, A^{0}=I$.) For $A$ in $J_{m}$ and $S \subseteq J_{m}$, we say that $A$ depends on $S$ if $A \leqq(\vee S) \vee Q^{m} M$ where we set $\bigvee \varnothing=0$. If we order the elements of $J_{m}$ by the lexicographic order of the exponent $n$-tuples, $\bigvee J_{m}$ is a minimal base for $Q^{m}$ if and only if for each $A$ in $J_{m}, A$ does not depend on the elements that precede it.

THEOREM 2.8. If $Q_{1}, \cdots, Q_{n}$ is a system of parameters for $(L, M)$, then $Q_{1}, \cdots, Q_{n}$ are analytically independent.

Proof. With notation as above, suppose that $m \geqq 1$ and $A$ is an element in $J_{m}$ such that $A$ depends on the set $\mathfrak{I}$ of elements that precede it in the lexicographic $n$-tuple order. Then for any $B$ in $J_{r}, B A \leqq B(\bigvee \mathfrak{I}) \vee$ $B Q^{m} M \leqq(\bigvee B \mathfrak{I}) \vee Q^{m+r} M$ where $B \mathfrak{I}=\{B C \mid C \in \mathfrak{I}\}$. So, $B A$ depends on the elements which precede it in $J_{m+r}$. Hence, if $q>m$ and $B$ is in $J_{q-m}$, then $B A$ can be deleted from $\bigvee J_{q}$ in forming a minimal base for $Q^{q}$ from the elements of $J_{q}$. There are $C((q-m)+(n-1), n-1)$ such $B A$ 's which can be deleted from $J_{q}$. Hence,

$$
\begin{aligned}
l\left(Q^{q} / Q^{q} M\right) \leqq & C(q+(n-1), n-1) \\
& -C((q-m)+(n-1), n-1) \text { for all } q>m .
\end{aligned}
$$

If $p(x)$ is the Hilbert polynomial for $Q$, this inequality implies that the degree of $p(x)$ is less than $n-1$. This contradiction proves the theorem. Q.E.D.

THEOREM 2.9. Let $(L, M)$ be a local Noether lattice satisfying the union condition. Then the following are equivalent:

(1) $M$ is a minimal reduction of $M$.

(2) Any minimal base for $M$ is analytically independent.

(3) $(L, M)$ is a regular local Noether lattice.

Proof. (1) implies (2). If $M=0$, the result is clear. If $M \neq 0$ is a principal element, then since $M^{n} M^{n+1}$ for any $n \geqq 1, M$ is analytically independent in $M$.

Suppose $M=M_{1} \vee M_{2} \vee \cdots \vee M_{d}$ where the $M_{i}$ are principal elements forming a minimal base for $M$ and $d \geqq 2$. Clearly, $l\left(M^{n} / M^{n+1}\right) \leqq C(n+d-1, d-1)$ for all $n \geqq 1$ since the products of degree $n$ in $M_{1}, \cdots, M_{d}$ join to form $M^{n}$. Since $L$ satisfies the union condition, by Lemma 2.4 , there exists an infinite collection of principal elements, $\left\{M_{i}\right\}$, such that any $d$ of them forms a minimal base for $M$. By Corollary 1.4, there exists an $(n, d)$-sequence $\Gamma$ in $L$ with factors from the $M_{i}$ 's of length $C(n+d-1, d-1)$. So, by Lemma $2.5, \Gamma$ can be extended to a minimal 
base for $M^{n}$. Hence, $l\left(M^{n} / M^{n+1}\right) \geqq C(n+d-1, d-1)$; and $M_{1}, \cdots, M_{d}$ are analytically independent.

(2) implies (3). Let $M_{1}, \cdots, M_{d}$ be a minimal base for $M$. Since the $M_{i}$ are analytically independent in $M, l\left(M^{n} / M^{n+1}\right)=C(n+d-1, d-1)$ for all $n \geqq 1$. Hence if $p(x)$ is the Hilbert polynomial for $M, p(n)=$ $C(n+d-1, d-1)$. From [5, 3.9], the altitude of $L$ is $d$. Therefore, $(L, M)$ is a regular local Noether lattice.

(3) implies (1). From Theorem 2.8, every regular system of parameters for $L$ is analytically independent. Hence, using the techniques in the proof of [7, §4, Theorem 1] we conclude that $M$ is a minimal reduction of $M$. Q.E.D.

From Theorem 2.9, we obtain a remark on $(n, d)$-sequences.

CoROllary 2.10. If $S^{*}$ and $S$ are as in $\S 1$, then the maximum length of an $(n, d)$-sequence in $S$ is $C(n+d-1, d-1)$.

Proof. Suppose there exists an $(n, d)$-sequence in $S$ of length $t>$ $C(n+d-1, d-1)$. Let $(L, M)$ be a regular local Noether lattice of altitude $d$ satisfying the union condition. Then by Theorem $2.9, M$ is a minimal reduction of $M$; and there exists an infinite sequence $\left\{M_{i}\right\}$ of principal elements such that any $d$ of them is a regular system of parameters for $L$. By Corollary 1.5 , there exists an $(n, d)$-sequence with factors in the $M_{i}$ 's of length $t$. This $(n, d)$-sequence can be extended to a minimal base for $M^{n}$. Consequently, $l\left(M^{n} / M^{n+1}\right) \geqq t>C(n+d-1, d-1)=l\left(M^{n} / M^{n+1}\right)$. Q.E.D.

\section{REFERENCES}

1. Garrett Birkhoff, Lattice theory, 3rd ed., Amer. Math. Soc. Colloq. Publ., vol. 25, Amer. Math. Soc., Providence, R.I., 1967. MR 37 \#2638.

2. K. P. Bogart, Structure theorem for regular local Noether lattices, Michigan Math. J. 15 (1968), 167-176. MR 37 \#2642.

3. A. H. Clifford and G. B. Preston, The algebraic theory of semigroups. II, Math. Surveys, no. 7, Amer. Math. Soc., Providence, R.I., 1967. MR 36 \#1558.

4. R. P. Dilworth, Abstract commutative ideal theory, Pacific J. Math. 12 (1962), 481-498. MR 26 \#1333.

5. E. W. Johnson, A-transforms and Hilbert functions in local lattices, Trans. Amer. Math. Soc. 137 (1969), 125-139. MR 38 \#5675.

6. - A-transforms in Noether lattices, Dissertation, University of California, Riverside, Calif., 1966.

7. D. G. Northcott, Ideal theory, Cambridge Tracts in Math. and Math. Phys., no. 42, Cambridge Univ. Press, Cambridge, 1953. MR 15, 390.

8. D. G. Northcott and D. Rees, Reductions of ideals in local rings, Proc. Cambridge Philos. Soc. 50 (1954), 145-158. MR 15, 596.

Department of Mathematics, Slippery Rock State College, Slippery Rock, PenNSYlVANia 16057 